\title{
NOTAS SOBRE LAS RELACIONES PREHISPÁNICAS ENTRE EL SALVADOR Y LA COSTA DE VERACRUZ, MÉXICO *
}

\author{
Luis Casasola \\ Centro de Estudios Maya \\ UNAM.
}

\section{Introducción}

$\mathrm{Al}$ analizar algunos aspectos de la arqueología salvadoreña, se ha visto que desde épocas muy antiguas aparecen objetos en El Salvador que señalan cierto tipo de relaciones con las culturas veracruzanas. Estas relaciones aparentemente se suceden hasta el Postclásico Tardío, y están íntimamente ligadas a través de la historia prehispánica con las migraciones de grupos conocidos en las fuentes históricas como pipiles.

En este trabajo se señalarán ciertas ideas que apuntan a un posible origen del culto de Xipe Tótec en El Salvador, como introducido por grupos del sur y centro de Veracruz, o de tradición cultural de estas zonas, durante el Clásico Tardío. En este proceso se introducen otros rasgos y elementos de la misma tradición costeña.

Durante el Postclásico, este culto se manifiesta como un producto probablemente de grupos inmigrantes, de origen o filiación cultural del Altiplano Central, que al mismo tiempo presentan ciertas características que los relacionan con elementos culturales de las tierras bajas del centro-sur de Veracruz y Tabasco.

\section{Representaciones de Xipe Tótec en El Salvador}

En un trabajo previo (Casasola, 1975), se reportaron dos efigies huecas de Xipe, de estilo típicamente Veracruz Central que aparentemente fueron encontradas en un lugar cercano al sitio Postclásico

* Una versión abreviada de este trabajo se presentó con el título: "El culto de Xipe Tótec en EI Salvador y su relación con la costa de Veracruz, México", en el $41^{\circ}$ Congreso Internacional de Americanistas, celebrado en la ciudad de México en septiembre de 1974. 
de Cihuatán, en el centro de El Salvador (Láms. 1-3). Estas figuras, comparándolas estilísticamente con piezas similares de México, fueron adscritas al Postclásico, posiblemente Tardío.

En ese mismo trabajo se mencionaba una figura de Xipe proveniente de la ribera oriental del Lago de Güija, en el departamento de Santa Ana. De este ejemplar solamente hemos podido obtener una fotografía proporcionada amablemente por el señor Tomás Vilanova, de Santa Tecla, El Salvador. Se puede ver que es una representación de Xipe Tótec en donde aparece sentado sobre una especie de trono o banco. Porta una máscara que le cubre el rostro, el cual emerge de un gran tocado en forma, posiblemente, de cipactli o serpiente. El cuerpo está cubierto con una piel de desollado que le cubre hasta las rótulas. Sobre los hombros presenta un elemento de forma irregular que descansa sobre ellos. En el pecho lleva un pectoral de forma circular, presentando debajo de éste una especie de chaleco. En la cintura porta un máxtlatl; en las muñecas lleva en cada lado tres pulseras o brazaletes; y sobre los brazos las que parecen ser unas ataduras. Por último, el personaje va calzado con sandalias (Lám. 4). ${ }^{1}$

Este estilo de figuras monumentales huecas conforma una gran tradición escultórica que es característica de las zonas centro y sur de Veracruz; apareciendo abundantemente en distintos sitios, con una temporalidad que va del Clásico Tardío al Postclásico; aun cuando su máximo apogeo ocurre durante el Clásico Tardío y Postclásico Temprano (Drucker, 1943: 57, 72 tabla 5; pls. 45-48; Medellín Zenil, 1960: Láms. 41, 53; McBride, 1971: 27, Láms. 107-115, 127, 128, 133-137; cf. especialmente con Lám. 107).

Un hacha con representación de Xipe (Lám. 5), desgraciadamente sin procedencia conocida, se encuentra en una colección particular en San Salvador. Es de roca volcánica, de color gris pálido, sin pulimento, y con las siguientes dimensiones: alto $21 \mathrm{~cm}$; ancho 17.5 $\mathrm{cm}$.; grueso $3.1 \mathrm{~cm}^{2}$ Los hallazgos de hachas en El Salvador son numerosos, algunos de los cuales ya han sido publicados anteriormente por varios investigadores (vid Casásola ob. cit.). Específicamente, otra hacha con efigie de Xipe ha sido publicada por Longyear (1944; pl. XII-20, 1966: 152, Fig. 12), procedente de Quelepa en el oriente del país, asignándola al Postclásico Temprano.

Stanley H. Boggs ha publicado otra representación monumental de Xipe en barro; procedente de la finca Santa Margarita, barrio

1 Borhegyi (1959) menciona este ejemplar pero no lo ilustra.

${ }^{2} \mathrm{La}$ fotografía y los datos correspondientes fueron proporcionados amablemente por Stanley H. Boggs. 
de Apaneca, en los límites con el cantón "El Cuje", aproximada. mente un kilómetro al este de Chalchuapa, departamento de Santa Ana (Boggs, 1945: 26, Figs. 4ª e-g; Casasola, ob. cit. Lám. 4). Adscribe su hallazgo como producto de las migraciones pipiles, y lo fecha hacia el Postclásico Tardío. Asimismo, el propio Boggs ha encontrado fragmentos de figuras huecas similares en estilo y técnica, aunque no idénticos, a la figura mencionada, en Tazumal, también en la zona de Chalchuapa (Boggs, ob. cit.: 29).

En el museo de Xalapa, Veracruz, se conserva una figura hueca de Xipe, procedente de Madereros, Municipio de Tlalixcoyan, la cual se fecha en el Postclásico Tardío y que es muy similar estilísticamente $y^{-}$en proporciones, al ejemplar de Chalchuapa. Boggs ha notado la similitud de este último con las piezas de Coatlinchan y Teotihuacán descritas por Saville y Linné respectivamente las cuales se ubican cronológicamente en el Postclásico, posiblemente Tardís (Boggs, ob. cit.: 29-30).

\section{El Culto de Xipe Tótec en Teotihuacán y Veracruz}

Con respecto al origen de este culto se han postulado varias teorías sin que hasta la fecha se haya dado una respuesta completamente satisfactoria (Coe, 1968: 111; Feuchtwanger, 1972: 69-72; Nicholson, 1972: 214-215; Reyna de Covarrubias et al, 1975: 3-4). Sin embargo, parece ser que su culto adquiere un mayor desarrollo en la costa de Veracruz durante el Clásico y el Postclásico, a pesar de que en esta última etapa, en su fase Tardía, las representaciones que existen del dios y las descripciones de los ritos asociados al culto, son abundantes en el Altiplano Central (Saville, 1929: 162; Linné, 1934: 83-86, Fig. 113; Sahagún, 1956: I-110-11, 142-149, III-65-67; Caso, 1964: Lám. IX; Dockstader, 1964: Fig. 23; Motolinía, 1971: 63).

En la época del contacto español el origen de esta deidad se situaba entre la zona yopi-tlappaneca de Oaxaca y Guerrero; y entre los zapotecas de Oaxaca (Seler, 1899 apud Nicholson 1972: 214; Caso y Bernal, 1952: 249).

En Teotihuacán en la fase Tzacualli, tenemos la representación del "Dios con máscara", que Caso y Armillas siguiendo a Seler, identifican con Xipe (Armillas, 1945: 20-21, Fig. 16 derecha; Caso, 1966: 269, Figs. 33a-b, 34a). Este tipo de representaciones aparece en figurillas de barro que son muy abundantes, y se suceden a través de la historia de la ciudad, hasta desembocar en el ejemplar más reciente que tenemos, el Xipe que encontró Linné en Xolalpan, perteneciente a la época Mazapan (Linné, 1934: 83-86, Fig. 113). 
También se conocen dos ejemplares grabados en piedra del "Dios con máscara" (Linné, 1942: Fig. 181; Armillas, 1945: 20, nota 68), los cuales recuerdan la representación de Xipe del Monumento 21 de Bilbao, y las dos esculturas similares de Aguná, en la zona de

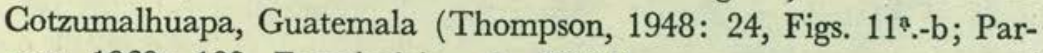
sons, 1969: 102, Frontispicio, pls. 30-31).

Las relaciones e intercambios entre la Costa del Golfo y Teotihuacán se inician desde una época muy temprana; inclusive, autores como Jiménez Moreno, han postulado que en la primera fase de Teotihuacán está presente un grupo costeño - Totonaca-Zoqueanojunto con los grupos autóctonos. Hay que recordar que Torquemada menciona que los totonacas construyeron las pirámides del Sol y la Luna (Jiménez Moreno, 1942: 140-141).

Medellín Zenil manifiesta que para fines del Preclásico hubo probablemente una migración de gentes que vivían entre los ríos Blanco y Papaloapan en Veracruz, hacia el Valle de México, combinándose ahí con los grupos existentes (Medellín Zenil, 1960: 118).

En la costa del Golfo las figurillas conocidas como Xipe-Tlazoltéotl aparecen en el Protoclásico. Especialmente en la zona semiárida Central Veracruzana se encuentra gran cantidad de ellas; teniendo su máximo desarrollo muy probablemente durante el Clásico Temprano (Ibid: 70, 75-76, Láms. 38-40 bis; Nicholson, 1971: 15, Láms. 116-118).

Es muy sugestivo que las figurillas en Teotihuacán que se han identificado como Xipes, y los ejemplares Xipe-Tlazoltéotl de la costa llevan como máscara el metz-xayacatl, es decir, una máscara hecha de la piel del muslo de un sacrificado. Las representaciones de desollamiento integral son más tardías en ambas zonas.

Para la zona del Totonacapan y sur de Veracruz, diversos autores han mencionado los estrechos lazos que existieron con Teotihuacán durante la época Clásica, y no vamos a referirnos a ellos ahora.

De igual forma, las investigaciones arqueológicas en otras áreas del sureste de Mesoamérica, como Oaxaca (Bernal, 1965: 802-804 Paddock, 1972: 237-238), Chiapas (Peterson, 1963: 124), Tierras Bajas Centrales (Thompson, 1939; Coe, 1972: 257-271), costa del Pacífico y Tierras Altas de Guatemala (Kidder, Jennings y Shook, 1946; Borhegyi, 1965: 3-58; Rands y Smith, 1965: 95-145; Shook, 1965: 180-194; Parsons, 1969: 156-169), así como en El Salvador (Boggs, 1962: 488-492, 1963: 505-507; Sharer, 1974: 172), muestran relaciones con la cultura teotihuacana en mayor o menor grado. 
Elementos, objetos y motivos que aparecen en El Salvador en distintas épocas, adscritos o relacionados a las culturas veracruzanas.

\section{Preclásico}

Varias piezas olmecas y olmecoides han aparecido en el oeste de El Salvador y han sido objeto de publicación (Boggs, 1950, 1971 y 1973; Coe, 1965: 767; Wassén, 1966; Casasola, 1974).

Sharer, con base en sus trabajos de investigación en la zona de Chalchuapa, considera que hubo un asentamiento olmeca en, o cerca, de esta área, cuyos fines eran comerciales y para controlar los productos locales: posiblemente hematita, cacao y obsidiana; dichos productos eran requeridos en la zona nuclear olmeca del sur de Veracruz y oeste de Tabasco. Estas relaciones, según Sharer, se manifiestan en la alfarería, figurillas y tal vez en la arquitectura (Sharer, 1974: 169-170). Es posible, entonces, que desde una fecha tan temprana como el Preclásico Medio existiera algún tipo de relaciones entre el occidente de El Salvador y el sur de Veracruz.

\section{Clásico}

Los hallazgos de hachas, yugos y palmas en el territorio salvadoreño están bien representados, localizándose en Quelepa, departamento de San Miguel, la única asociación hasta ahora conocida de los tres elementos en Mesoamérica (Andrews V, 1970: figs. 3-5, 7-10).

En Tazumal, en la zona de Chalchuapa, se han encontrado 3 hachas y 3 yugos (Boggs, 1945a: 33-36, 1950: 270, 1963: 506). Asimismo, 2 palmas incompletas de la misma zona, y otra íntegra, de los alrededores de la Laguna Cuzcachapa, se conservan en la colección del señor Tomás Vilanova en Santa Tecla, La Libertad.

En Campana-San Andrés se reporta también el hallazgo de un yugo y un hacha (Boggs, 1943: Fig. 2b, 1950: 265). De la hacienda Tasagero, en la zona de Suchitoto, departamento de Cuscatlán, proviene otra hacha, la cual fue publicada por Lothrop (1926: Fig. 50). De igual forma, Spinden (1915: 480), menciona el hallazgo de un yugo liso en Apaneca, departamento de Ahuachapán.

Este tipo de objetos aparece principalmente, en el occidente y centro del país, adscribiéndose al Clásico Tardío. Estas zonas, de acuerdo con las fuentes históricas, eran territorios pipil al tiempo de la Conquista (Lothrop, 1945: 134; Thompson, 1948: 11, 1970: map 3; Miles, 1957).

Por otro lado, también aparecen en El Salvador figurillas con rue- 
das, especialmente en dos zonas: Cihuatán, en el centro del país, y Quelepa, en el oriente. En la primer área se considera que pertenecen al Postclásico, posiblemente Tardío. Las de Quelepa, al Clásico Tardío y Postclásico Temprano (Boggs, 1973a: 62).

Revisando los trabajos hasta ahora publicados sobre el tema, se ve que tal vez la mayor concentración de este tipo de piezas ocurre en el centro y sur de Veracruz durante el Clásico y Postclásico Temprano, más aun que en el Altiplano Central (Ibid: Fig. 26). Este mismo autor, siguiendo a Von Winning, ve ciertos indicios en cuanto a que los ejemplares salvadoreños se derivan de los mexicanos; v considera, con base en que no se han encontrado hasta el momento en Chiapas y Guatemala, que pudieron ser transportados por mar, posiblemente desde las costas de Guerrero y Oaxaca hacia El Salvador "llegando durante el Periodo Clásico a los esteros orientales de lo que son ahora las costas de los departamentos de Usulután y San Miguel, y en tiempos posteriores en la Barra de Santiago (especialmente: Isla de El Cajete) y otros lugares costeros del extremo suroccidental del país" (Ibid: 62-63).

En Quelepa, en la fase Shila hacia 500 d.C., aparecen los primeros tipos de alfarería polícroma los cuales son de pasta fina, delgada, de color naranja; por lo general con engobe blanco, y con un ligero baño naranja y pintura roja y negra. Las formas más comunes son cajetas de paredes redondeadas, y vasijas en forma de pera. La decoración es a base de diseños geométricos y figuras de animales.

Este tipo de alfarería se prolonga hasta la fase Lepa, que ve el abandono del sitio; aproximadamente por 1000 a 1100 d.C. (Andrews V, 1970: 27).

Las cerámicas de pasta fina tienen su mayor concentración en las tierras bajas del sur de Veracruz, Tabasco y norte de Chiapas; siendo seguramente esta zona de donde se dispersan a otras áreas de Mesoamérica. Andrews V (comunicación personal, 1974) ve una fuerte influencia de la costa del Golfo sobre El Salvador Oriental durante el Clásico Tardío; la cual se manifiesta en el complejo de yugos-hachas-palmas, los juegos de pelota, instrumentos musicales, y especialmente la alfarería, señalando que ésta es de pasta fina durante este período en la zona centro-este de El Salvador, muy similar a la cerámica Naranja Fino de los grupos Balancán (Z) y Altar (Y).

Personalmente, analizando las colecciones del Museo Nacional de El Salvador, vimos algunas piezas con estas características, pero en realidad no son muy abundantes.

Por otro lado, hay una gran ocurrencia de instrumentos musicales 
de viento en territorio salvadoreño, los cuales, tienen una amplia distribución en el tiempo y en el espacio, apareciendo prácticamente en todo el país. Existe una gran diversidad de clases y formas. Recientemente, Boggs (1974) ha analizado 310 ejemplares que clasifica en tres categorías: flautas, flautas-silbatos, y silbatos. La mayoría de ellos tienen una temporalidad que cae en el Clásico Tardío.

Andrews V (1973: Figs. 1-3) ha publicado 3 flautas procedentes de Quelepa, las cuales son muy similares a ejemplares de Nopiloa y otros lugares de Veracruz. Una de estas flautas es de pasta fina, clasificada dentro del tipo Obrajuelo Plain, que es el tipo doméstico más característico de Quelepa en el Clásico Tardío. Andrews V considera que éste, junto con otros rasgos que ya se han mencionado, muestran una fuerte influencia de la costa del Golfo en El Salvador en esta época (Ibid: 10).

Tal diversidad de formas, clases y decoraciones en instrumentos musicales de viento, sólo es comparable en Veracruz durante el Clásico. José Luis Franco considera que en esta última zona es en donde se encuentra la mayor diversidad de instrumentos musicales en la América Prehispánica, ocurriendo esto durante el Clásico (Franco, 1971: 18).

\section{Postclásico}

En esta época, como ya se mencionó, posiblemente en la fase Tardía, aparecen figurillas con ruedas en el sitio de Cihuatán y en la Isla de El Cajete; en el centro y occidente del país respectivamente. En el primer sitio se encuentran asociadas a fragmentos de braseros monumentales de paredes gruesas y decoración al pastillaje, de formas bicónicas o de "reloj de arena". Algunos de ellos llevan efigies modeladas que representan a Tláloc. Asimismo se encuentran vasijas, tapaderas y soportes de incensarios con la efigie del Dios de la Lluvia; efigies de jaguares sentados (Cf. Drucker, 1943: pl. 48k, 1); cajetes trípodes de color negro muy bien pulido; fragmentos de sahumerios tipo sartén con mangos huecos y cilíndricos; cajetes y ollas polícromas rojo, negro y naranja sobre engobe blanco, las cuales están representadas también en la isla El Cajete en el departamento de Ahuachapán, apareciendo asociadas ahí con piezas del "tipo" Mixteca-Puebla (Lothrop, 1927: Fig. 15b; Cf. Druker, 1943: pls. 24, 38; Longyear, 1944: pl. XIII-9; Boggs, 1973a-51-54, Figs. 15-25).

Fragmentos de grandes braseros con decoración al pastillaje han aparecido también en Tazumal y Campana-San Andrés, encontrán- 
dose en ambos lugares en la superficie, señalando la última etapa de ocupación (Boggs, 1944: 70, 1950: 273).

El único ejemplar hasta ahora conocido de figurillas con ruedas en la isla de El Cajete, es una cabeza de venado (?) que perteneció probablemente al cementerio Postclásico Tardío de este sitio. En este cementerio es muy alta la ocurrencia de vasijas de un tipo similar al Mixteca-Puebla; considerando Boggs que inclusive algunas de ellas son importaciones directas de México (Boggs, 1973a: Fig. 20b, nota 12).

En el sitio arqueológico conocido como Pueblo Viejo, en la Hacienda "Las Marías", departamento de la Libertad en el centro del país, aparece una serie de grandes ollas funerarias con asas, las cuales contienen como ofrenda algunos de los objetos siguientes: vasijas con efigie de Tláloc, figurillas planas hechas en molde de tipo Mazapa; pequeñas cabezas de figurillas de tipo "toltecoide"; sahumerios tipo sartén con mangos huecos y cilíndricos; pequeños vasos en forma de copa con soporte pedestal de barro café sin engobe; pequeños cajetes trípodes de barro café sin engobe; figuras huecas de sapos; y cajetes simples polícromos en rojo, negro y blanco sobre naranja; y negro y blanco sobre naranja rojizo (Casasola en preparación; Cf. Lothrop, 1927: Figs. 15b, 16; Weber, 1922: Figs. 17a-b). Asimismo, se encuentran braseros monumentales muy similares a los de Cihuatán, Campana-San Andrés y Tazumal.

Los cajetes polícromos mencionados recuerdan a tipos polícromos del Altiplano Mexicano; y ocurren piezas como éstas en otros lugares: Isla El Cajete que ya se ha señalado; Campana San Andrés y Nejapa, en el occidente y centro de El Salvador respectivamente (Boggs, 1950: 264, nota 10; Longyear, 1944: pl. XI-10).

Las características arquitectónicas y cerámicas de Pueblo Viejo, hacen suponer que pertenece al Postclásico Tardío; sin que se pueda saber si existen etapas anteriores por falta de investigación.

Berlin ha publicado algunos vasos en forma de copa de pasta Naranja Fino U que se ubica, en el Horizonte Cintla (1300-1500 d.C.) y que proceden de Atasta, Campeche, en la zona al noroccidente de la laguna de Términos, y de Tamulté de las Sabanas, Tabasco, en la margen occidental del río Grijalva (Berlin, 1956: Figs. 7b, 11e). Estos ejemplares son muy similares a los encontrados en Pueblo Viejo.

Por otro lado, Drucker (1943: pls. 24, 29-31, 39 tabla 8) ilustravasijas Tláloc, figurillas "toltecoides", y figurillas planas moldeadas tipo Mazapa, de Cerro de las Mesas; muy similares a los ejemplares provenientes de la Hacienda "Las Marías". Especialmente las figuri- 
llas Mazapa presentan esta particularidad. Drucker asigna estas piezas como pertenecientes al periodo Cerro de las Mesas Superior I ((1000-1300 d.C.), (Ibid: 73, 80-81, Fig. 210).

De sitios cercanos a Quetzaltepeque, también en el departamento de La Libertad; provienen otros ejemplares de tipo Mazapa, los cuales se conservan en distintas colecciones en El Salvador. No está de más insistir en que esta zona así como el occidente del país, era de lengua pipil en el siglo XVI.

Algunos comentarios sobre las migraciones pipiles a Centroamérica, especialmente a El Salvador.

Borhegyi (1965) se ha referido a 3 migraciones que se suceden en varias épocas y que llegan a distintos lugares de América Central. La primera de ellas la situa en el Clásico Medio (400-500 d.C.), y está constituida por grupos teotihuacanos que hacia esta época se asien$\tan$ entre otros lugares, en distintas zonas de Veracruz, Guerrero, costa del Pacífico y Altos de Guatemala.

Esta migración tuvo según este autor, carácter comercial y religioso. Entre los elementos diagnósticos que se pueden reconocer para este fenómeno se encuentran: alfarería naranja delgado; vasijas Tláloc; vasos trípodes cilíndricos; candeleros y vasijas florero.

Esos grupos emigrantes paulatinamente fueron absorbidos por las poblaciones nativas, o empujados por otros pueblos migratorios hacia áreas marginales, hacia los fines de Teotihuacán. (Ibid: 39-40, Fig. 4).

Ya se han mencionado anteriormente las intensas relaciones entre la cultura teotihuacana y la costa del Golfo durante esta época.

Parsons (1969), al referirse a las características culturales que definen la fase Laguneta del Clásico Medio en Bilbao, especialmente al hablar de los elementos asociados al complejo del juego de pelota, menciona que son introducidos a la región de Cotzumalhuapa desde la costa del Golfo. Entre ellos se reconocen hachas, yugos, lápidas con distintas escenas de sacrificios humanos, representaciones de dioses ceestiales, elementos vegetales, aves, seres esqueléticos, etc. Asimismo, se reconocen varios rasgos que tienen su origen en Teotihuacán y Oaxaca.

Todos estos elementos se van a combinar para dar forma a un estilo y cultura denominada Cotzumalhuapense, la cual se va a desarrollar en esta etapa, llegando hasta la fase Santa Lucía del Clásico Tardío (Parsons, ob. cit.: 72-73, 77, 149, 156; pls. 20a-e, 21a-b, 28, 30-34, 42c-d, 61a-c).

La figura grabada del monumento 21 de este sitio que se ha iden- 
tificado como posible Xipe, se ubica dentro de la fase Laguneta. De igual forma, las dos esculturas de Xipe procedentes de Aguná, en la misma región de Cotzumalhuapa, probablemente se ubican en el Clásico Tardío.

García de León (1969: 4-5, 7-8, 38) al referirse a la presencia nahua en la costa del Golfo, menciona que fue probablemente debido al proceso de expansión y dispersión de los teotihuacanos probablemente por 800 d.C., o tal vez un poco antes. Citando a Swadesh (1954-55) señala que la diversificación del nahua empieza alrededor de 600 d.G.; que coincide con el apogeo de Teotihuacán.

Por otro lado, la posible representación de Xipe del monumento 21 de Bilbao, aparece asociada a figuras de granos de cacao con efigies antropomorfas. Esta zona de la costa del Pacífico fue una de las mayores regiones productoras del grano de acuerdo con las fuentes, al igual que la parte occidental costera de El Salvador.

Como ya se ha visto anteriormente, desde época Preclásica existen asentamientos de grupos afiliados a la costa del Golfo, en la zona de Chalchuapa; para controlar supuestamente, entre otras cosas, la producción de dicha planta (Sharer, 1974: 169-170).

Esta región es muy favorable desde el punto de vista de las comunicaciones entre la costa y las Tierras Altas de Guatemala. Estamos de acuerdo con Parsons cuando considera que las intrusiones de grupos foráneos en la zona de Cotzumalhuapa son, probablemente, con el objeto de controlar las tierras productoras de cacao de la costa del Pacífico (1969: 149).

La fase Esperanza de Kaminaljuyú, tan estrechamente relacionada con Teotihuacán, podría ser mejor entendida en el sentido de un control político ejercido sobre el primer sitio por la metrópoli del Altiplano Central. Esto daría probablemente entre otras cosas, un control sobre la zona productora de cacao de las tierras bajas de la costa, ya que Kaminaljuyú tiene una excelente ubicación para el acceso a estas últimas, tal como lo han señalado Sanders y Price (1968: 168).

$\mathrm{La}$ aparición de elementos culturales de otras zonas en las distintas partes de Mesoamérica Clásica, está señalando el amplio nivel de intercambio de ideas, productos y relaciones de diverso tipo entre los distintos grupos de esta época.

Borhegyi (ob. cit.: 40) se refiere a una segunda migración pipil que llama "Pipiles-Tajinizados-Teotihuacanos" (Pipil-Nicarao), los cuales eran grupos que a la caída de Teotihuacán, después de 650 d.C. se desplazan a lugares como Azcatpotzalco, Cholula y Xochi- 
calco. Otros emigran hacia las tierras bajas de la costa del Golfo y se agregan a las colonias existentes de inmigrantes teotihuacanos más tempranos. En esta zona adoptan algunos de los elementos típicos de la cultura de las tierras bajas, como el culto a las cabezas trofeo y el sacrificio de la decapitación asociado al juego de pelota (Stirling, 1943 apud Borhegyi: 40). Esta migración ocurre entre 700 y 900 d.C. aproximadamente.

Entre los elementos diagnósticos que señalan este fenómeno se encuentran: alfarerías Plumbate San Juan y Robles, Tiquisate Naranja Fino $\mathrm{Z}$ y $\mathrm{Y}$, yugos, hachas y marcadores del juego de pelota de distintas formas, etc. (Ibid: 39).

Jiménez Moreno (1959), basándose en Torquemada, ha señalado una migración de grupos de tradición teotihuacana los cuales estaban asentados en Cholula y sus alrededores, los que debido al dominio a que estaban sujetos por un grupo de olmecas históricos, emigran hacia Centroamérica, pasando por la región de los Tuxtlas, y de ahí a los "despoblados de Soconusco", asediados siempre por los olmecas. Este autor considera que el fenómeno se ubica por 800 d.C. aproximadamente, y la ocurrencia de hachas y yugos en las distintas partes del sureste de Mesoamérica, señalaría el paso de estos grupos inmigrantes (Jiménez Moreno, 1959: 1077-1088).

Sin embargo, estamos de acuerdo con Parsons al considerar que los elementos escultóricos asociados al juego de pelota, habían sido introducidos al este de Mesoamérica (él prefiere usar el término América Media) probablemente más temprano (Parsons, ob. cit.: 185).

En lugares del Usumacinta como Altar de Sacrificios y Seibal, se ve en el fin del Clásico la irrupción de patrones culturales no autóctonos. Especialmente en Altar de Sacrificios, la fase Jimba (900-980 d.C. aprox.) presenta una drástica discontinuidad e intrusión en los patrones cerámicos. Esto es debido, según Adams, a la presencia de grupos provenientes de las tierras bajas del sur de Veracruz, Tabasco y Chiapas, los cuales ocupan esos sitios del Usumacinta (Adams, 1971: 5, 163; 1973: 148).

En esta fase, las clases cerámicas de Altar de Sacrificios son de pasta fina. Uno de los tipos más característicos es Pabellón Moldeado-Esculpido (Pabellón Molded-Carved), que se incluye dentro del grupo Naranja Fino Altar (Y).

Pabellón muestra ciertas afinidades estilísticas generales con el estilo tolteca, y con los desarrollos cerámicos de la costa de Veracruz '(Adams, 1971: 50-51, 136, 164; 1973: 148). 
La gente que ocupa Altar de Sacrificios y Seibal en esta época, es considerada de carácter eminentemente militarista; el cual es patente en la alfarería, figurillas y estelas (Thompson, 1970: 38-43, pls. 1-2; Adams, 1971: 162-165).

Estos grupos pudieron contribuir al derrumbe de los sitios Clásicos a lo largo del Usumacinta y sus tributarios, como de hecho se ve en Seibal. Al reconstruir su movimiento desde las tierras bajas del Golfo, se piensa que pasan por la zona al este de Bonampak; por el Usumacinta hacia el sureste de Yaxchilán y Piedras Negras; desde ahí hacia el Usumacinta Superior, para llegar a Altar de Sacrificios (Adams, 1971: 163-165).

Hay que recordar que en lugares como Palenque y Copán se encuentran yugos después de que estos sitios dejan de registrar fechas calendáricas en sus monumentos (Jiménez Moreno, 1959: 1089).

Aunque en Altar y Seibal no se han encontrado yugos, es muy probable que las gentes que introducen esos elementos foráneos en Copán y Palenque, estén relacionados de alguna forma con los que ocupan Altar y Seibal.

Estos dos sitios ofrecen particularmente, una ubicación geográfica muy propicia para el control e intercambio con otras zonas. La región de Altar de Sacrificios controla tres sistemas fluviales: el Río Salinas corre hacia las tierras altas; el Pasión cruza la parte sur de las tierras bajas Centrales; y el Usumacinta que desemboca hacia la Costa del Golfo. Por otro lado, el Río Lacantún que drena las tierras bajas de Chiapas nororiental, no queda muy retirado (Thompson, 1970: 39-40; Adams, 1971: 163).

Lehmann en una fecha más temprana ya se había referido a estas posibles rutas (1920: 1062).

Parsons (1969: 167-168) al referirse a la difusión del estilo Clásico Veracruzano hacia el sur de Mesoamérica, en los fines del Clásico, menciona los vasos de tecali con elementos tajinoides encontrados en Uaxactún y en el Valle del Ulúa. Señala una ruta de difusión mayor que pudo haber cruzado la parte sur del Petén, desde Palenque y Toniná, hacia Quirigua y Copán en el este. Elementos como representaciones de Tláloc, signos del año a la manera teotihuacana, y guerreros con escudos rectangulares aparecen en Piedras Negras y Yaxchilán.

En Quelepa, El Salvador, los elementos culturales atribuidos a la costa del Golfo, aparecen en una fecha tan temprana como 500 d.C., en la fase Shila; mientras que el hallazgo asociado de hacha- 
yugo-palma, se fecha hacia el fin del Clásico, como ya se ha visto (Andrews V, 1970: 27-28, 30, 32).

En Tazumal, Boggs (1950: 270-271) ha notado que existe un hiatus en la construcción, entre el fin del Clásico y el Postclásico Temprano, que dura aproximadamente 200 años. Los hallazgos de hachas y yugos ocurren en tumbas del Clásico Tardío o Final, por lo que creemos que ese lapso puede estar relacionado con la llegada de grupos foráneos, con elementos culturales veracruzanos.

En Asunción Mita, para regresar a Guatemala, se ha encontrado un yugo liso y 3 esculturas zoomorfas asociadas a los juegos de pelota de ese sitio (Strömsvik, 1950: Lám. 7a-d). En Guaytán, en los alrededores de San Agustín Acasaguastlán, se ven ciertos indicios según Smith y Kidder, que apuntan para una posible migración de grupos nahuas hacia los fines del Clásico; y se considera que este movimiento pudo traer como consecuencia el abandono de Guaytán y otros lugares de la cuenca del Motagua Medio (Smith y Kidder, 1943: 179).

Hay que recordar que estas dos zonas eran de hablantes de pipil en el siglo XVI, de acuerdo a los cronistas españoles (vid mapa 3 en Thompson, 1970).

La tercera migración pipil que considera Borhegyi se ubica entre 1000 y 1200 d.C.; y la llama Nonoalca-Pipil-Tolteca-Chichimeca. Estaba compuesta por gente del norte de México que supuestamente hablaban náhuatl, y que habían abandonado Tula en el Estado de Hidalgo, bajo el mando de Ce-Acatl-Topiltzin-Quetzalcóatl. Después de pasar por Cholula, llegan a la costa del Golfo, a lugares de Veracruz, Tabasco y Campeche. Esto sucede alrededor del año 1000 d.C.

El otro grupo que compone esta migración estaba constituido por los nonoalcas, que aparentemente eran una mezcla de hablantes de náhuat, o Pipiles-Tajinizados-Teotihuacanos, quienes habían permanecido en la zona de la Laguna de Términos después de que sus parientes se habían movido hacia el sur, aproximadamente entre 700 y 800 d.C.; y un grupo de gentes teotihuacanas que habían salido de Cholula por la misma época; y que habían estado sujetos al dominio de los olmecas históricos, estableciéndose en la zona de la desembocadura del Río Usumacinta, y en la región de la Laguna de Términos. En esta zona se mezclan con los Pipiles-Tajinizados-Teotihuacanos o "Pipil-Nicarao".

Según Borhegyi, hacia la primera mitad del siglo XI, los toltecachichimeca se mezclan con los grupos residentes nonoalca-pipiles y chontales; y algún segmento de esta unión emigra hacia Chichén 
Itza, Uxmal y Mayapán en la Península de Yucatán. Otros más siguen el Río Usumacinta y sus tributarios, hacia las tierras altas de Guatemala (Borhegyi, 1965: 40-41).

Esta opinión está de acuerdo con lo que sostiene Carmack para explicar el poblamiento de la Altiplanicie Guatemalteca, hacia 1250 d.C., por grupos de tradición cultural no maya, que llegan desde la región alrededor de la Laguna de Términos (Carmack, 1970: 66$67,70,86)$. De igual forma se mencionan las posibles rutas de intercambio desde Tabasco y otros lugares de las tierras bajas del Golfo, a través del Usumacinta y sus tributarios, para llegar a las tierras altas de Guatemala, el Petén, y al Valle del Motagua (Ibid: 60).

Ya hemos visto que estas rutas probablemente existían desde épocas más tempranas. Por otro lado, se ve que las cerámicas diag. nósticas del Postclásico Temprano en las tierras altas, con la excepción del Plumbate Tohil, indican una influencia de la costa del Golfo (Borhegyi, 1965: 47).

Ya se ha señalado la semejanza entre la cerámica del Postclásico de sitios como Cihuatán, Pueblo Viejo, Tazumal, Isla El Cajete, y otros lugares en El Salvador, con la cerámica de lugares del sur de Veracruz y Tabasco.

Boggs (1949) ha publicado un grupo de braseros Tláoc, los cuales caen en el Postclásico Tardío, y que proceden de los alrededores de Quetzaltepeque, en el centro de El Salvador. Estos braseros presen$\tan$ una gran semejanza tanto en técnica de manufactura, estilo y dimensiones, con uno de Veracruz ilustrado por McBride, el cual pertenece al mismo periodo (1971: 27, Lám. 126).

El mismo Boggs (1963a), al analizar un pequeño lote de piezas procedentes de la ribera occidental del Lago de Güija en Guatemala, llega a la conclusión de que fueron hechas por gente tolteca llegada de México, o con tradiciones culturales de ese carácter. Las ubica hacia el siglo XIV de nuestra era.

Nosotros agregaríamos que también se ve cierto parentesco con productos de Veracruz (Cf. Boggs, ob. cit., especialmente Figs. 2b, 3a, con Drucker, 1943; pls. 45b-b' y 48f, respectivamente).

$\mathrm{Al}$ realizar un viaje de reconocimiento en varios sitios de la zona del Güija, pudimos ver que probablemente existió una gran densidad de población en épocas antiguas, de acuerdo a la cantidad y carácter de los sitios recorridos en esa área.

En uno de los lugares reconocidos, Igualtepeque (Longyear, 1944: 21, Figs. 9, 13, pls. XIII-1, 3-4, 6; Jiménez, 1959-60), se encuentran gran cantidad de petrograbados con distintos temas, siendo los 


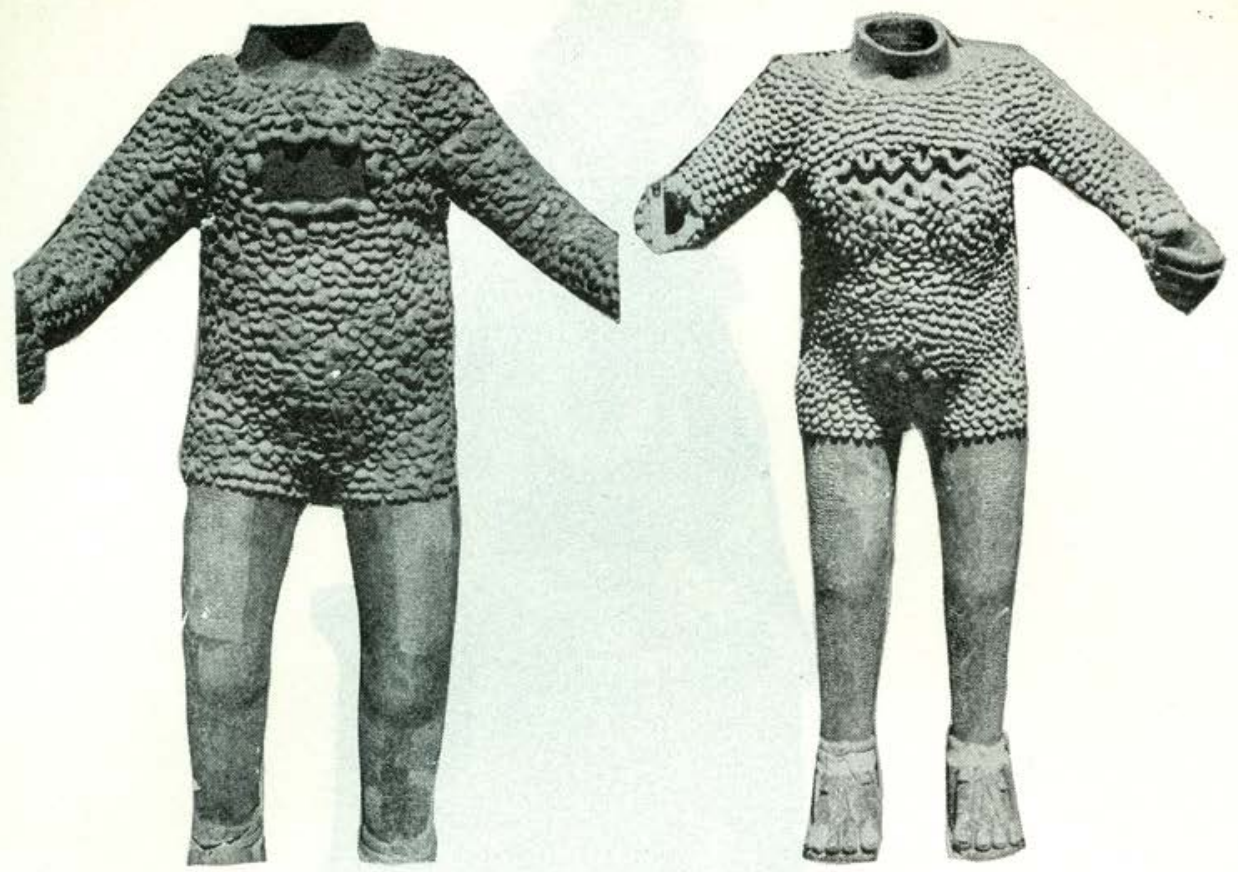

Láminas 1, 2 y 3. Figuras de Xipe Totec.

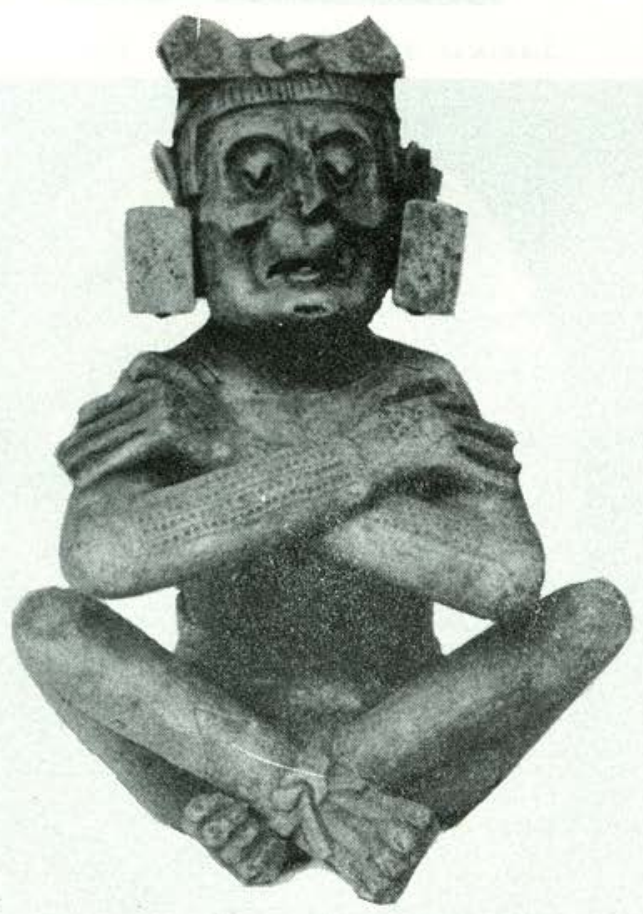

Estudios de Cultura Maya. Vol. X, 1976/7

Instituto de Investigaciones Filológicas/ Facultad de Filosofía y Letras Centro de Estudios Mayas, UNAM 


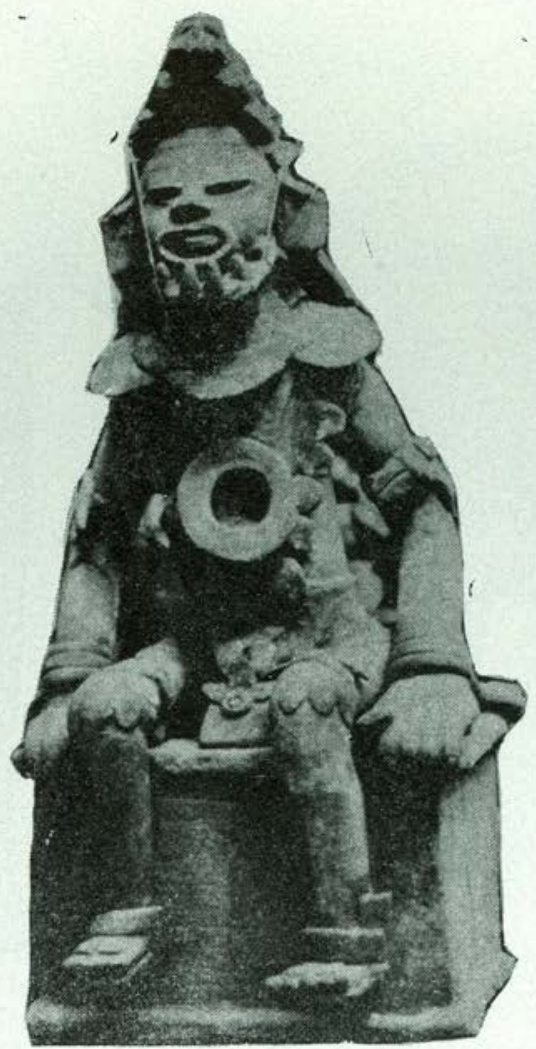

LÁminas 4 y 5 . Figuras de Xipc.



Estudios de Cultura Mãya. Vol, X, 1976/7

Instituto de

Centro de Estudios Mayas, UNAM

http://www.iifilologicas.unam.mx/estculmaya/ 
más abundantes representaciones de serpientes emplumadas, discos solares, figuras de monos, figuras antropomorfas estilizadas, y diseños más convencionales y esquemáticos.

En la finca Potosí, sitio reconocido por Longyear (ob. cit.: 22) se localizan otros petrograbados que recuerdan a los de Igualtepeque. La finca Potosí también se encuentra en el departamento de Santa Ana.

Desgraciadamente, no se ha hecho ninguna excavación en Igualtepeque que ayude a fechar los monumentos antes mencionados.

En algunos lugares de Veracruz como Pilapan Mirador, municipio de Soteapan, se encuentran algunos petrograbados, los cuales, recuerdan de alguna forma a los de Güija. (vid. Medellín Zenil, 1960 a: Láms. 10, 12).

En los alrededores de Izalco, departamento de Sonsonate, El Salvador, en sitios arqueológicos como Huiscoyolate, San José Huiscoyolate, etc., se encuentran petrograbados que representan principalmente figuras zoomorfas: monos, caracoles, guajalotes (?), etc.; figuras solares y otras representaciones simbólicas. Las figuras solares aparecen asociadas a varios diseños que podrían ser quizá signos calendáricos. Esto no se pudo constatar realmente, porque la visita que se hizo fue muy breve.

En Huiscoyolate se recolectaron algunos tiestos y vasijas completas, entre las cuales habían varias de alfarería Copador Polícromo, que es un buen marcador del Clásico Tardío en el occidente y centro del país. Esto no quiere decir que los monumentos grabados pertenezcan a esta época, sino que creemos que se ubican en una fecha más tardía.

Vivó ha señalado la asombrosa repetición de toponímicos del sur de Veracruz, suroeste y costa de Chiapas, que aparecen especialmente en El Salvador (1970: 27-31).

La tradición recogida por Torquemada, menciona que los pipiles después de abandonar los "despoblado de Soconusco", llegan a $Y z$ cuintlan (Escuintla), Eçalcos (Izalco), y Mictlan (Mita); para posteriormente llegar a Nicaragua, en donde son conocidos como nicaraos. Ahí se establecen en la región del Istmo de Rivas, junto al Lago de Nicaragua (Lehmann, 1920: 1060; Lothrop, 1926a: 5, 8; Thompson, 1941: 33-34, 54; Jiménez Moreno, 1959: 1077; Torquemada, 1969: 331-333; León Portilla, 1972: 27, 30-33).

$\mathrm{Al}$ analizar los petrograbados de Ometepe, Isla El Muerto, y otros lugares en el gran Lago de Nicaragua (Matilló Vila, 1965, 1968, 1973), podemos ver que algunos recuerdan muy estrechamente a los 
de Igualtepeque. Aquellas islas se ubican en el norte del Istmo de Rivas, este último el hogar tradicional de los nicaraos.

Lothrop al estudiar la alfarería de tipo "Incisa debajo del engobe", que aparece en distintas partes del sur de Nicaragua, piensa que está relacionada a algunos tipos que se encuentran en el sur de Veracruz (1926a: 193, pls. LXXXIII-LXXXVII).

\section{Comentarios finales}

Es tiempo ya de plantear algunas hipótesis en cuanto a lo que se ha venido manejando.

Se ha podido ver que en distintas épocas de la historia precolombina mesoamericana hubo una serie de movimientos de pueblos con distintas características: comerciales, militares, religiosas, los cuales produjeron una serie de intercambio de ideas, motivos y elementos culturales. Estos son perceptibles a través de los datos arqueológicos, por medio de las fuentes históricas, la evidencia lingüística, y aún por el registro etnográfico.

En ciertos casos se puede hablar realmente de movimientos de grupos o migraciones; en otros de intercambio de objetos y temas; en algunos más, de adopción de elementos foráneos; y ciertamente en otros - tal vez la mayoría - de transmisión únicamente de ideas y conceptos, más no de objetos.

$\mathrm{El}$ grado de seguridad con que la arqueología puede detectar cualquiera de estos procesos es incierto y vago.

El Salvador por su posición geográfica, ha demostrado con base en los datos antropológicos, que funcionó como un corredor o puente a través del cual pasaron en uno y otro sentido, objetos e ideas de Meso y Sudamérica en el transcurso del tiempo. Especialmente aquí se presenta una encrucijada a nivel de punto crítico en cuanto a discernir qué es lo Meso y qué es lo Sudamericano.

Las relaciones con Veracruz, específicamente las partes centro y sur, parecen ser especialmente fuertes a partir del Clásico Tardío. Creemos que en esta época se introducen y adoptan en El Salvador distintos rasgos de esa zona, entre ellos el culto a Xipe Tótec.

Las rutas de difusión pudieron ser varias, de algunas de las cuales tal vez no tengamos idea, solamente más adelante, con más investigaciones es posible que podamos tener una visión más certera.

Una ruta a través de la cual se transmitieron estas ideas, pudo seguramente ser la que proponen Parsons y Adams, a través del Usumacinta y sus tributarios hacia las tierras altas de Guatemala y la parte 
sur de las tierras bajas Centrales; y de ahí hacia la costa del Pacífico, y Valle del Motagua y Golfo de Honduras respectivamente (Thompson, 1970: 39-40).

Esta ruta probablemente es muy antigua. Otra seguramente fue a través del Istmo de Tehuantepec hacia la costa de Chiapas y Guatemala, y de ahí a El Salvador y otro lugares de Centroamérica. Navarrete (1973) entre otros, se ha referido a esta última.

En el Postclásico, el intercambio de ideas y relaciones de distinto carácter se mantiene entre El Salvador y Veracruz Meridional. La arqueología, la lingüística y la etnohistoria así lo señalan.

Durante el apogeo de Tula, a la caída del llamado Imperio Tolteca, y aún después, estas relaciones e intercambios se siguen manifestando.

Boggs, al final de uno de sus últimos trabajos en donde se refiere a este problema, señala: "Sin embargo, por razones todavía no bien comprendidas, parece que El Salvador antiguo mantuvo contactos culturales esporádicos a través de unos 20 siglos antes de la Conquista con las poblaciones de Veracruz central y norteño..." (1973a: 64).

La existencia en El Salvador de lugares como Nahuizalco, Apaneca e Izalco, que aún hoy en día tienen hablantes de náhuat o pipil, nos indica claramente que es debido a la migración de grupos mayores; de otra forma no se explica este fenómeno.

Los estudios de léxico-estadística apuntan a la posibilidad de que antes del siglo XII existía una continuidad de grupos nahuas, desde el sur de Veracruz, Tabasco, hasta Guatemala y El Salvador, incluyendo los Altos de Chiapas (García de León: 34-35).

Creo realmente, que sólo a partir de un enfoque combinado en el que intervengan la arqueología, lingüística, etnohistoria y otras disciplinas, podemos llegar a tener un mejor conocimiento de este tipo de problemas.

\section{BIBLIOGRAFIA}

Adams, Richard E. W.

1971 The Ceramics of Altar de Sacrificios, Papers of the Peabody Museum of Archaeology, 63-1, Harvard University, Cambridge.

1973 "Maya Collapse: transformation and termination in the ceramic sequence at Altar de Sacrificios", The Classic Maya Collapse: 133-163, Patrick Culbert ed. The School of American Research, University of New México Press, Alburquerque. 
ANDREWS, V, E. WYLLYS

1970 "Excavations at Quelepa, eastern El Salvador", Ceramica de Cultura Maya et al. 6: 21-40, Temple University, Philadelphia.

1973 Flautas precolombinas procedentes de Quelepa, El Salvador, Dirección de Publicaciones del Ministerio de Educación. San Salvador.

Armmlas, Pedro

1945 "Los dioses de Teotihuacán", Reimpresión de Anales del Instituto de Etnologia Americana, VI: 35-61, Universidad Nacional de Cuyo, Mendoza.

BerLin, Heinrich

1956 "Late pottery horizons of Tabasco, Mexico", Contributions to American Anthropology and History, 59-606: 95-153, Carnegie Institution of Washington, Washington.

Bernal, Ionacio

1965 "Archaeological synthesis of Oaxaca", Handbook of Middle American Indians, 3: 788-813, R. Wauchope general ed., University of Texas Press, Austin.

Boggs, Stanley H.

1943 "Notas sobre las excavaciones en la hacienda "San Andrés", departamento de La Libertad", Tzunpame, III-3: 104-126, Museo Nacional, San Salvador.

1944 "Excavations in Central and Western El Salvador", Appendix $\mathrm{C}$ en Archaeological Investigations in El Salvador, Memoirs of the Peabody Museum of Archaeology and Ethnology, IX-2: 53-72, Harvard University, Cambridge.

1945 "Comentarios sobre una estatua de barro hallada en la zona arqueológica de Chalchuapa", Tzunpame, V-4: 26-32, Museo Nacional, San Salvador.

1945a "Informe sobre la tercera temporada de excavaciones en las ruinas de "Tatzumal", Tzunpame, V-4: 33-45, Museo Nacional, San Salvador.

1949 "Tláloc incensarios in the "Baratta collection" El Salvador", Notes on Middle American Archaeology and Ethnology, 394: 36-45, Carnegie Institution of Washington, Division of Histori cal Research, Cambridge.

1950 "Archaeological excavations in El Salvador", For the Dean. Essays in Anthopology in honor of Byron Cummings on his eighty ninth birthday: 259-276, Hohokam Museums Association y The Southwestern Monuments Association, Tucson y Santa Fe.

1950a "Olmec" pictorgraphs in the Las Victorias group, Chalchuapa zone, El Salvador", Notes on Middle American Archaeology 
and Ethnology, 4-99: 85-92, Carnegie Institution of Washington, Division of Historical Research, Cambridge.

1962 "Excavations at Tazumal, El Salvador", Yearbook American Philosophical Society: 488-492, Philadelphia.

1963 "Excavations at Tazumal, El Salvador". Yearbook American Philosophical Society: 505-507, Philadelphia.

1963a "Apuntes sobre varios objetos de barro procedentes de Los Guapotes, en el Lago de Güija, Antropología e Historia de Guatemala, XV-1: 15-27, Instituto de Antropología e Historia de Guatemala, Guatemala.

1971 "An Olmec mask-pendant from Ahuachapan, El Salvador", Archaeology, 24-4: 356-358, Archaeological Institute of America, New York.

1973 "Vestimentas y tocados anteriores a los Mayas", Américas, 25-2: 19-24, Secretaría General de la O.E.A., Washington.

1973a "Figurillas con ruedas de Cihuatán y el oriente de El Salvador", Cultura, 59: 37-74. Dirección de Publicaciones del Ministerio de Educación, Salvador.

1974 "Notes on Pre-Columbian wind instruments from El Salvador", Baessler-Archiv, n.f., XXII: 23-71, Berlin.

Borhegy, Stephan F. de

1959 "Underwater archaeology in Guatemala", Actas del XXXIII Congreso Internacional de Americanistas, II: 229-240, San Jos!.

1965 "Archaeological Synthesis of the Guatemalan Highlands", Handbook of Middle American Indians, 2: 3-58, R. Wauchope general ed., University of Texas Press, Austin.

Carmack, Robert M.

1970 "Toltec influence on the Postclassic culture history of Highland Guatemala", Archaeological Studies in Middle America, 26: 49-92, Middle American Research Institute, Tulane University, New Orleans.

Casasola, Luis

1974 "Una figurilla olmeca de Coatepeque, Santa Ana, El Salvador", Notas Antropológicas, I-18, Instituto de Investigaciones Antropológicas, UNAM, México.

1975 "Dos figuras de Xipe T...tec en El Salvador", Balance $y$ perspectiva de la antropología de Mesoamérica y del norte de México, vol. 2 de arqueología: 143-153, XIII Mesa Redonda de la Sociedad Mexicana de Antropología, México.

s.f. "Notas sobre algunas piezas del Postclásico Tardío, procedentes del departamento de La Libertad, El Salvador". (en preparación).

\section{Caso, Alfonso}

1964 The Aztecs. People of the sun, University of Oklahoma Press, Norman. 
1966 "Dioses y signos teotihuacanos", Teotihuacán, primera parte: 249-279, XI Mesa Redonda de la Sociedad Mexicana de Antropolog|a, México.

Caso, Alfonso e Ignacio Bernal

1952 Urnas de Oaxaca, Memorias del Instituto Nacional de Antropología e Historia, II. México.

Coe, Mrghaet. D.

1965 "The Olmec style and its distributions", Handbook of Middle American Indians, 3: 739-775, R. Wauchope general ed., University of Texas Press, Austin.

1968 America's first civilization, American Heritage Publishing Co. Inc. New York.

Coe, Wrlliam R.

1972 "Cultural contact between the Lowland Maya and Teotihuacan as seen from Tikal, Peten, Guatemala", Teotihuacán, segunda parte: 257-271, XI Mesa Redonda de la Sociedad Mexicana de Antropología, México.

Dockstader, F. J.

1964 Indian art in Middle America. New York Graphic Society, Greenwich.

Drucker, Philip

1943 Ceramic stratigraphy at Cerro de Las Mesas, Veracruz, Mexico, Bull 141, Bureau of American Ethnology, Smithsonian Institution, Washington.

Feuchtwanger, Franz

1972 "Representaciones relacionadas al culto de Xipe procedentes de Tlatilco y sitios similares", Religión en Mesoamérica: 6972, XII Mesa Redonda de la Sociedad Mexicana de Antropología, México.

Franco, José Luis

1971 "Musical instruments from Central Veracruz in Classic times", Ancient art of Veracruz: 18-22, The Ethnic Art. Council of Los Ángeles.

García de León, Antonio

1969 Pajapan. Una variante del Nahua del Este, Tesis Profesional de Maestría, ENAH, México.

Jiménez, Tomás Fidias

1959- "Reflexiones sobre las inscripciones hundidas en el Lago de 1960 Güija", Anales del Museo Nacional David J. Guzmán, IX33-34: 11-15, Ministerio de Cultura, San Salvador. 
JimÉnez MoReno, W.

1942 "El enigma de los Olmecas", Cuadernos Americanos, V-5: 113-145, México.

1959 "Síntesis de la Historia Pretolteca de Mesoamérica", Esplendor del México Antiguo: 1019-1108, Centro de Investigaciones Antropológicas de México, México.

Kidper, A. V. J. D. Jennings y E. M. Shook.

1946 Excavations at Kaminaljuyú, Guatemala, Pub. 561, Carnegie Institution of Washington, Washington.

Lehmann, WaLter

1920 Zentral-Amerika. Die Sprachen Zentral-Amerikas in ihren beziehungen zueinander sowie su Süd-Amerika und Mexiko, Band II, Berlin.

León Portilla, Miguel

1972 Religión de los Nicaraos. Análisis y comparación de tradiciones culturales Nahuas, Serie de Cultura Núhuatl, Monografías: 12, Instituto de Investigaciones Históricas, UNAM, México.

Linné, Sigvald

1934 Archaeological researches at Teotihuacan, México, Ethnographical Museum of Sweden, n.s. 1. Stockholm.

1942 Mexican Highlands Cultures. Archaeological researches at Teotihuacan, Calpulalpan and Chalchicomula in 1934/35, The Ethnographical Museum of Sweden, n.s. 7 Stockholm.

LONGYEAR, JOHN M.

1944 Archaeological investigations in El Salvador, Memoirs of the Peabody Museum of Archaeology and Ethnology, IX-2 Harvard University, Cambridge.

1966 "Archaeological survey of El Salvador", Handbook of Middle American Indians, 4: 132-156, R. Wauchope general ed., University of Texas Press, Austin.

Lothrop, SAMUel K.

1926 "Stone sculptures from the finca Arevalo, Guatemala", Indian Notes, 3-3: 147-171, Museum of the American Indian, Heye Foundation, New York.

1926a Pottery of Costa Rica and Nicaragua. 2 vols., Contributions from the Museum of the American Indian, Heye Foundation, New York.

1927 "Pottery types and their sequence in El Salvador", Indian Notes and Monographs, 1-4: 165-220, Museum of the American Indian, Heye Foundation, New York.

1945 "La frontera sud-oriental de los Mayas", Tzunpame, V-4: 132-141, Museo Nacional, San Salvador. 
Matillo Vila, Joaquín

1965 Estas piedras hablan. Estudio preliminar del Arte Rupestre en Nicaragua, Editorial Hospicio, Managua.

1968 El Muerto, isla santuario. Estudio de su arte rupestre, Imprenta Nacional, Managua.

1973 Ometepe, isla de círculos y espirales, Centro de Investigaciones Rupestres, Managua.

MCBRIDE, Harold, W.

1971 "Figurine types of Central and Southern Veracruz", Ancient Art of Veracruz: 23-30, The Ethnic Art Council of Los Angeles, Los Angeles.

Medellín Zenil, Alfonso

1960 Cer micas del Totonacapan, Universidad Veracruzana, Xalapa.

1960a "Monolitos inéditos Olmecas", La Palabra y el Hombre, 16: 75-97, Universidad Veracruzana, Xalapa.

MrLes, S. W.

1957 The Sixteenth Century Pokom-Maya; a documentary analysis of social structure and archaeological setting, Transactions of the American Philosophical Society, 47-4, Philadelphia.

1965 "Sculpture of the Guatemala-Chiapas Highlands and Pacific slopes, and associated hieroglyphs", Handbook of Middle Ame. rican Indians, 2: 237-275, R. Wauchope general ed., University of Texas Press, Austin.

Motolinía, Fray Toribio de Benavente

1971 Memoriales o Libro de las Cosas de la Nueva España y de los Naturales de ella, Edmundo O'Gorman ed., Instituto de Investigaciones Históricas, UNAM, México.

Navarrete, Carlos

1973 "El sistema prehispánico de comunicaciones entre Chiapas y Tabasco", (Informe preliminar), Anales de Antropologia, $\mathrm{X}$ : 33-92, Insituto de Investigaciones Históricas, UNAM, México.

Nicholson, Henry B.

1971 "The iconography of Classic Central Veracruz ceramic sculptures", Ancient Art. of Veracruz: 13-17, The Ethnic Art Council of Ios Angeles, Los Angeles.

1972 "The cult of Xipe Totec in Mesoamerica", Religión en Mesoamérica: 213-218, XII Mesa Redonda de la Sociedad Mexicana de Antropología, México.

\section{PADDOCK, JOHN}

1972 "El ocaso del Clásico", Teotihuacán, segunda parte: 141-147, XI Mesa de la Sociedad Mexicana de Antropología, México. 
Parsons, Lee A.

1969 Bilbao, Guatemala an archaeological study of the Pacific coast Cotzumalhuapa region, Vol. 2, publications in anthropology, 12, Milwaukee Public Museum, Milwaukee.

Peterson, Fredrick A.

1963 Some ceramics from Mirador, Chiapas, México, Papers the New World Archaeological Foundation, 15, Brigham Young University, Provo.

RANds, Robert L. y Robert E. Smith

1965 "Pottery of the Guatemalan Highlands", Handbook of Middle American Indians, 2: 95-145, R. Wauchope general ed., University of Texas Press, Austin.

Reyna de Covarrubias, Rosa M. et al.

1975 "Posibles representaciones de deidades en figurillas Preclasicas del Altiplano", Balance y perspectiva de la antropología de Mesoamérica y del norte de México, Vol. 2 de arqueología: 221-230, XIII Mesa Redonda de la Sociedad Mexicana de Antropología, México.

Sanders, Wimlinams T. y Bárbara J. Price

1968 Mesoamerica. The evolution of a civilization, Randon House Inc., New York.

Sahagun, Bernardino de

1956 Historia General de las Cosas de la Nueva España, Ángel M. M. Garibay ed., 4 vols. Editorial Porrúa, México.

Saville, Marshall $\mathrm{H}$.

1929 "The aztecan god Xipe Totec", Indian Notes, 6-2: 151-174, Museum of the American Indian, Heye Foundation, New York.

Sharer, Robert J.

1974 "The prehistory of the southeastern Maya periphery", Current Anthropology, 15-2: 165-187, The University of Chicago Press, Chicago.

Shoox, Edwin M.

1965 "Archaeological survey of the Pacific coast of Guatemala", Handbook of Middle American Indians, 2: 180-194, R. Wauchope general ed., University of Texas Press, Austin.

SMrth, A. L. y A. V. KIDDER

1943 "Explorations in the Motagua Valley, Guatemala", Contributions to American Anthropology and History, 41-546: 101 182, Carnegie Institution of Washington, Washington. 
Spínden, Herbert J.

1915 "Notes on the archaeology of Salvador", American Anthropologist, n.s. 17-3: 446-487, Lancaster.

Stromsvik, Gustav

1950 "Las ruinas de Asunción Mita. Informe de su reconocimiento", Antropología e Historia de Guatemala, II-1: 23-29, Instituto de Antropología e Historia de Guatemala, Guatemala.

Swadesh, Mauricio

1954- "Algunas fechas glotocronológicas importantes para la prehis-

1955 toria nahua", Revista Mexicana de Estudios Antropológicos, 14: 173-192, Sociedad Mexicana de Antropología, México.

Thompson, ERIC J. S.

1939 Excavations at San José, British Honduras. Pub. 506 Carnegie Institution of Washington, Washington.

1941 "Dating of Certain inscriptions of non Maya origin", Theoretiacl approaches to problems, 1, Carnegie Institution of Washington, Washington.

1948 "An archaeological reconnaissance in the Cotzumalhuapa region, Escuintla, Guatemala", Contributions to American Anthropology and History, 44-574, Carnegie Institution of Washington, Washington.

1970 Maya history and religion, University of Oklahoma Press, Norman.

Torquemada, Juan de

1969 Monarquía Indiana, Introducción de Miguel León Portilla, 3 vols, Editorial Porrúa, México.

Vivo Escoto, Jorge A.

1970 "El poblamiento náhuat en El Salvador y otros países de Cenamérica", Anuario de Geografía, X: 9-43, Universidad Nacional Autónoma de México, México.

WASSÉn, Henry S.

1966 "An olmec stone figurine from Ahuachapan, El Salvador". Ethnos, 1-4:84-89. The Etnographical Museum of Sweden, Stockholm.

WEBER, FRIEDRích

[1922] "Zur archaologie Salvadors", Festschrift Eduard Seler: 619. 644, Stuttgart.

(Las secciones consultadas de Lehmann, 1920; fueron amablemente traducidas por el prof. Manfred Reinhold, a quien doy mi más expresivo agradecimiento). 